\title{
COMPUTATION OF THE ZEROS OF $p$-ADIC $L$-FUNCTIONS. II
}

\author{
R. ERNVALL AND T. METSÄNKYLÄ
}

\begin{abstract}
The authors have carried out a computational study of the zeros of Kubota-Leopoldt $p$-adic $L$-functions. Results of this study have appeared recently in a previous article. The present paper is a sequel to that article, dealing with the computation of the zeros under certain conditions that complicate the original situation.
\end{abstract}

\section{INTRODUCTION}

This paper continues the authors' computational study, begun in [2], of the zeros of the Kubota-Leopoldt $p$-adic $L$-functions $L_{p}(s, \chi)$. We will discuss four themes, all of which came up but were left aside in [2].

In the following, the article [2] is referred to as Part I. When referring to an individual section or proposition of that paper we write, say, $\S \mathbf{I} .3$ or Proposition I.5. We retain the basic notation of Part I; in particular, $p$ is an odd prime, $f_{\theta}(T)=\sum_{j=0}^{\infty} a_{j} T^{j}$ denotes the Iwasawa power series determined by the first factor $\theta$ of $\chi$, and $\lambda=\lambda_{\theta}$ stands for the $\lambda$-invariant of this power series.

Our first question, considered in $\S 2$, concerns the computation of the zeros of $L_{p}(s, \chi)$ when the second factor of $\chi$ is nonprincipal. The remaining sections deal with the computation of the zeros $T_{1}, \ldots, T_{\lambda}$ of $f_{\theta}(T)$ under certain conditions that complicate the original situation discussed in Part I. Thus, in $\S 3$ there are two zeros $T_{1}$ and $T_{2}$ close to each other, $\S 4$ introduces types of the Newton polygon of $f_{\theta}(T)$ different from our two basic types, and the final $\S 5$ shows how to compute zeros $T_{k}$ lying in wildly ramified extensions of $\mathbb{Q}_{p}$.

A sample of numerical results related to each of these sections is given in Tables VI-X at the end of the paper.

We recall that a crucial step in the computation of a zero $T_{0}$ of $f_{\theta}(T)$ is the determination of the extension $E=\mathbb{Q}_{p}\left(T_{0}\right)$ and of an initial approximation $t_{0}$ of $T_{0}$ satisfying the condition

$$
f_{\theta}\left(t_{0}\right) \equiv 0 \quad\left(\bmod \pi^{2 \gamma+1}\right),
$$

where $\pi$ is a prime element of $\mathscr{O}_{E}$ and $\gamma=v_{\pi}\left(f_{\theta}^{\prime}\left(T_{0}\right)\right)$. To accomplish this, one first reduces the possible candidates for $E$ to a family of relatively few fields and expands $T_{0} \pi$-adically with unknown coefficients; then $E$ and $t_{0}$ will be

Received by the editor April 30, 1992.

1991 Mathematics Subject Classification. Primary 11S40, 11Y05, 11Y40, 11R20, 11 R23.

Key words and phrases. p-adic $L$-functions, computation of zeros, factorization of polynomials, Newton's tangent method, Abelian fields, Iwasawa theory. 
obtained by solving successively a sequence of approximation congruences

$$
f_{\theta}\left(T_{0}\right) \equiv 0 \quad\left(\bmod \pi^{m}\right), \quad m=1,2, \ldots, m_{0},
$$

denoted by $\mathscr{C}\left(\pi^{m}\right)$ below.

When describing the computation of $T_{0}$, we usually assume, to fix ideas, that $a_{0}=f_{\theta}(0)$ does not vanish. If $a_{0}=0$, the procedure is essentially the same; one just excludes the zero $T_{0}=0$, which is known to be simple, and then replaces $f_{\theta}(T)$ by $f_{\theta}(T) / T$. In this case we say that $f_{\theta}(T)$ is of type $Z$.

\section{L-FUNCTIONS INVOLVING CHARACTERS OF THE SECOND KIND}

In this section we are concerned with the zeros $s_{0}$ of $L_{p}\left(s, \theta \psi_{n}\right)$, where $\psi_{n}$ is a nonprincipal character of the second kind. Recall that $\psi_{n}$ is of order $p^{n}$ and conductor $p^{n+1} \quad(n \geq 1)$. We will discuss the questions of how to find examples of $L_{p}\left(s, \theta \psi_{n}\right)$ having a zero $s_{0}$, and how to compute this $s_{0}$.

Suppose we have found a zero $T_{0}$ of some Iwasawa power series $f_{\theta}(T)$. By $\S$ I.3, if there is a corresponding zero $s_{0}$ of some $L_{p}\left(s, \theta \psi_{n}\right)$, then $s_{0}=$ $\log \left(1+T_{0}\right) / \log (1+d p)$ and $v_{p}\left(T_{0}\right)=1 /(p-1) p^{n-1}$. In the range of our computations, the only $p$-ordinals of $T_{0}$ of this form are $v_{3}\left(T_{0}\right)=1 / 2, v_{5}\left(T_{0}\right)=1 / 4$, and $v_{3}\left(T_{0}\right)=1 / 6$, and the respective values of $\lambda$ are 2,4 , and 6 (or 3,5 , and 7 when $f_{\theta}(T)$ is of type $\left.Z\right)$. Since, moreover, the coefficients of $f_{\theta}(T)$ in all examples are from $\mathbb{Z}_{p}$, Proposition I.5(i) shows that $T_{0}$ must belong to $\mathbb{Q}_{p}\left(\zeta_{p^{n}}\right)$ in order that $s_{0}$ lie in $D_{s}$, the domain of definition of $L_{p}(s, \chi)$.

The case $v_{3}\left(T_{0}\right)=1 / 6$, which leads to a wildly ramified extension $\mathbb{Q}_{3}\left(T_{0}\right)$, will be studied in $\S 5$. We found no example of this kind with $T_{0} \in \mathbb{Q}_{3}\left(\zeta_{9}\right)$.

As for the other two cases, it follows from Proposition I.5(ii) that the condition $T_{0} \in \mathbb{Q}_{p}\left(\zeta_{p}\right)$ is even sufficient for $s_{0}$ to be a zero of a $L_{p}\left(s, \theta \psi_{1}\right)$. We have the following practical criterion.

Proposition 1. Let $\lambda=p-1$ and $v_{p}\left(a_{0}\right)=1$, so that $v_{p}\left(T_{0}\right)=1 /(p-1)$. Then $T_{0} \in \mathbb{Q}_{p}\left(\zeta_{p}\right)$ if and only if $a_{01}=a_{\lambda 0}$.

Proof. The Newton polygon of $f_{\theta}(T)$ is of the "first type" discussed in $\S$ I.9. From that discussion it is seen that $T_{0} \in \mathbb{Q}_{p}(\sqrt[p-1]{r p})$, where $r$ is determined by the conditions

$$
\begin{gathered}
T_{0} \equiv x \pi \quad\left(\bmod \pi^{2}\right), \quad 0<x<p, \\
a_{01}+a_{\lambda 0} r x^{p-1} \equiv 0 \quad(\bmod p) .
\end{gathered}
$$

Since $\mathbb{Q}_{p}\left(\zeta_{p}\right)=\mathbb{Q}_{p}(\sqrt[p-1]{-p})$, the assertion follows.

For $p=5$ our numerical material contains five examples with $\lambda=4$ and $v_{5}\left(a_{0}\right)=1$, and one similar example of type $Z$. In all these, $T_{0} \notin \mathbb{Q}_{5}\left(\zeta_{5}\right)$. Four of the examples are exhibited in Tables IV and V of Part I; the remaining two are $(5,5708,0)$ and $(5,-12712,1)$.

Our main case is that of $p=3, \lambda=2$, and $v_{3}\left(a_{0}\right)=1$. Applying the criterion of Proposition 1 to the tables computed in Program A (Part I), one finds numerous examples of this case which give zeros of $L_{3}\left(s, \theta \psi_{1}\right)$. Including some analogous cases with $\lambda=3$, we settled 13 examples of this kind completely.

Before describing the computation, we observe that there are two characters $\psi_{1} \bmod 9$, say $\psi_{+}$and $\psi_{-}$, identified by the equations $\psi_{ \pm}(1+3 d)=-\frac{1}{2} \pm \frac{\pi}{2}$, where $\pi=\sqrt{-3}$. A zero $T_{0}$ with $v_{3}\left(T_{0}\right)=1 / 2$ may be written in the form $T_{0}=3 b+c \pi$, where $b, c \in \mathbb{Z}_{3}, c \not \equiv 0(\bmod 3)$. Then, by Proposition I.3, the 
number $s_{0}=\log \left(1+T_{0}\right) / \log (1+3 d)$ corresponding to $c \equiv+1(\bmod 3)$ is a zero of $L_{3}\left(s, \theta \psi_{+}\right)$, in fact, the only zero of this function (under the assumption that $\lambda=2)$. Its conjugate is of course the zero of $L_{3}\left(s, \theta \psi_{-}\right)$.

After computing $L_{3}(s, \theta), f_{\theta}(T), T_{0}$, and $s_{0}$ in the usual way, we proceed with the computation of the function

$$
L_{3}\left(s, \theta \psi_{1}\right)=\sum_{i=0}^{\infty} u_{i}^{\prime} s^{i} .
$$

As in $\S I .5$, this means the approximation of $u_{i}^{\prime}$ by rational integers congruent to $u_{i}^{\prime}$ modulo a prescribed power $p^{M}$; this makes sense since $u_{i}^{\prime} \rightarrow 0$ as $i \rightarrow \infty$. Here we make use of Washington's formula with $\Phi=9 d$ in the way described in $\S \S I .5$, I.11. A lower bound for $v_{p}\left(u_{i}^{\prime}\right)$ needed in the computation is provided by Proposition 2 below.

Recall that

$$
L_{p}(s, \theta \psi)=f_{\theta}\left(\rho(1+d p)^{s}-1\right),
$$

where $\rho=\psi(1+d p)^{-1}, \psi=\psi_{n}$. To check the correctness of our $L_{3}\left(s, \theta \psi_{1}\right)$, we evaluated both sides of this formula for $s=1$. As a final check it was verified that $L_{3}\left(s_{0}, \theta \psi_{1}\right)$ vanishes $\bmod \pi^{M_{1}}$, where (see the end of $\S I .11$ )

$$
M_{1}=\min \left(2 M, \quad M-3+\min _{i>0} v_{\pi}\left(u_{i}^{\prime}\right)\right) .
$$

By the remark at the end of the present section, $M_{1} \geq M$.

Example. Let $\theta$ be the character defined by $(3,-1147,1)$. The following table presents $L_{3}(s, \theta)$ (1st column) and $f_{\theta}(T)$ (3rd column) according to the same principles as the Tables $\mathrm{I}-\mathrm{V}$ in Part $\mathrm{I}$; see $\S \mathrm{I} .10$ for a description. The middle column lists the coefficients $u_{0}^{\prime}, \ldots, u_{6}^{\prime}$ of $L_{3}\left(s, \theta \psi_{ \pm}\right)$; here, $\pi=$ $\sqrt{-3}$. The coefficients $u_{i}^{\prime}$ with $i>6$ are zero to the accuracy displayed in this column.

\begin{tabular}{|l|l|l|}
\hline 0.11000 & $0.0200 \pm 0.0122 \pi$ & 0.11000 \\
0.01122 & $0.0111 \pm 0.1102 \pi$ & 0.1102 \\
0.01020 & $0.0110 \pm 0.0002 \pi$ & 1.100 \\
0.00011 & $0.0001 \pm 0.0220 \pi$ & 0.20 \\
0.00102 & $0.0011 \pm 0.0000 \pi$ & 2.0 \\
0.00000 & $0.0000 \pm 0.0001 \pi$ & 0. \\
0.00011 & $0.0001 \pm 0.0000 \pi$ & \\
\hline
\end{tabular}

The zeros of $f_{\theta}(T)$ are $T_{1,2}=0.10 \pm 2.0 \pi$ (approximately), and the corresponding numbers $s_{1,2}=0.0 \pm(2$. $) \pi$ are the zeros of $L_{3}\left(s, \theta \psi_{\mp}\right)$. (The upper and lower signs correspond to each other.)

Table VI contains further examples of this kind. In Table VIa there are two similar examples with an additional pair of zeros of $f_{\theta}(T)$ and $L_{3}(s, \theta)$.

We conclude this section by proving the following estimate (cf. Proposition I.6).

Proposition 2. For any $\psi=\psi_{n}(n \geq 1)$, let $L_{p}(s, \theta \psi)=\sum_{i=0}^{\infty} u_{i}^{\prime} s^{i}$. Then

$$
v_{p}\left(u_{i}^{\prime}\right) \geq i-\frac{i}{p-1} \quad(i=0,1, \ldots) .
$$


Proof. Considering the right-hand side of (1), we write

$$
\rho(1+d p)^{s}-1=\rho \exp (s \log (1+d p))-1=\sum_{i=0}^{\infty} d_{i} s^{i},
$$

where $d_{0}=\rho-1$ and $d_{i}=\rho(\log (1+d p))^{i} / i$ ! for $i \geq 1$. This gives the expansions

$$
\left(\rho(1+d p)^{s}-1\right)^{j}=\sum_{i=0}^{\infty} d_{i j} s^{i} \quad(j=0,1, \ldots)
$$

with $d_{00}=1, d_{i 0}=0 \quad(i \geq 1)$, and

$$
d_{i j}=\sum_{t_{1}+\cdots+t_{j}=i} d_{t_{1}} \cdots d_{t_{j}} \quad(i \geq 0, j \geq 1) .
$$

We show that

$$
v_{p}\left(d_{i j}\right) \geq j v_{p}(\rho-1)+\left(1-\frac{1}{p-1}\right) i \quad(i \geq 0, j \geq 0) .
$$

This is trivial for $j=0$; so let $j \geq 1$. Denote by $j^{\prime}$ the number of positive indices in a set $\left\{t_{1}, \ldots, t_{j}\right\}$ with $t_{1}+\cdots+t_{j}=i$. For simplicity of notation, assume that the positive indices are just $t_{1}, \ldots, t_{j^{\prime}}$, so that

$$
\sum_{\nu=1}^{j} v_{p}\left(d_{t_{\nu}}\right)=\left(j-j^{\prime}\right) v_{p}(\rho-1)+\sum_{\nu=1}^{j^{\prime}} v_{p}\left(d_{t_{\nu}}\right)
$$

Since $v_{p}(\rho-1) \leq 1 /(p-1)$ and $v_{p}\left(d_{t_{\nu}}\right)=t_{\nu}-v_{p}\left(t_{\nu} !\right) \geq t_{\nu}-\left(t_{\nu}-1\right) /(p-1)$ $\left(\nu=1, \ldots, j^{\prime}\right)$, we obtain the estimate

$$
\sum_{\nu=1}^{j} v_{p}\left(d_{t_{\nu}}\right) \geq j v_{p}(\rho-1)+\left(1-\frac{1}{p-1}\right) \sum_{\nu=1}^{j^{\prime}} t_{\nu}
$$

This proves (2).

Equation (1) now allows us to write

$$
\sum_{i=0}^{\infty} u_{i}^{\prime} s^{i}=\lim _{J \rightarrow \infty} \sum_{j=0}^{J} a_{j} \sum_{i=0}^{\infty} d_{i j} s^{i}=\lim _{J \rightarrow \infty} \sum_{i=0}^{\infty}\left(\sum_{j=0}^{J} a_{j} d_{i j}\right) s^{i},
$$

whenever $s \in D_{s}$. Restrict $s$ for a moment to the subset of $D_{s}$ defined by $v_{p}(s)>0$. Then it follows, in view of (2), that

$$
\sum_{i=0}^{\infty} u_{i}^{\prime} s^{i}=\sum_{i=0}^{\infty}\left(\sum_{j=0}^{\infty} a_{j} d_{i j}\right) s^{i}
$$

(see Lemma in [3, p. 53]). Hence,

$$
u_{i}^{\prime}=\sum_{j=0}^{\infty} a_{j} d_{i j} \quad(i=0,1, \ldots),
$$

and this together with (2) implies the proposition. 
Remark. Let, in particular, $\lambda=p-1$ and $v_{p}\left(a_{0}\right)=1$. Then the coefficients of the series for $L_{p}\left(s, \theta \psi_{1}\right)$ satisfy the stronger inequality

$$
v_{p}\left(u_{i}^{\prime}\right) \geq i-\frac{i}{p-1}+1 \quad(i=0,1, \ldots) .
$$

This is seen from (3) and (2) upon observing that $v_{p}\left(a_{j}\right) \geq 1$ for $j=0, \ldots$, $p-2$ and $j v_{p}(\rho-1) \geq 1$ for $j \geq p-1$. In the range of our computations, $v_{p}\left(u_{i}^{\prime}\right)$ attains this lower bound for most values of $i$.

\section{TWO ZEROS CLOSE TO EACH OTHER}

This section continues our study of the case $\lambda=2, v_{p}\left(a_{0}\right)=2$ in which the Newton polygon of $f_{\theta}(T)$ is of the "second type" considered in $\S$ I.9. This case was settled under the assumption that the two zeros $T_{1}$ and $T_{2}$ (denoted by $T_{0}^{\prime}$ and $T_{0}^{\prime \prime}$ in $\left.\S I .9\right)$ satisfy $v_{p}\left(T_{1}-T_{2}\right)<2$. We now describe how to deal with examples in which $v_{p}\left(T_{1}-T_{2}\right)$ is bigger. As before, it is assumed throughout that $T_{1} \neq T_{2}$.

We begin with the following proposition that will also be needed in $\S 4$.

Proposition 3. If all the zeros $T_{1}, \ldots, T_{\lambda}$ of $f_{\theta}(T)$ are pairwise distinct, then

$$
v_{p}\left(f_{\theta}^{\prime}\left(T_{k}\right)\right)=\sum_{\substack{j=1 \\ j \neq k}}^{\lambda} v_{p}\left(T_{k}-T_{j}\right) \quad(k=1, \ldots, \lambda) .
$$

Proof. By the $p$-adic Weierstrass preparation theorem (see $\S \mathbf{I} .2$ ),

$$
f_{\theta}(T)=u_{\theta}(T) w_{\theta}(T)
$$

where $u_{\theta}(T)$ is an invertible power series and $w_{\theta}(T)$ a monic polynomial of degree $\lambda$ having $T_{1}, \ldots, T_{\lambda}$ as its zeros. Differentiate this equation to obtain $f_{\theta}^{\prime}\left(T_{k}\right)=u_{\theta}\left(T_{k}\right) w_{\theta}^{\prime}\left(T_{k}\right)$ and, hence, $v_{p}\left(f_{\theta}^{\prime}\left(T_{k}\right)\right)=v_{p}\left(w_{\theta}^{\prime}\left(T_{k}\right)\right)$.

Going back to the above case, suppose that the rational integers $x, y$, and $r$ fulfil the conditions

$$
\begin{gathered}
0<x<p, \quad 0 \leq y<p, \quad r=1 \text { or } g(\text { a primitive root of } p), \\
T_{1,2} \equiv x p \pm y p \sqrt{r p} \quad\left(\bmod p^{2}\right)
\end{gathered}
$$

Such numbers are found in the way presented in $\S$ I.9. In particular, if $y=0$, then $v_{p}\left(T_{1}-T_{2}\right) \geq 2$, and we may put

$$
T_{k} \equiv x p+z_{k} p^{2} \quad\left(\bmod p^{5 / 2}\right) \quad(k=1,2),
$$

where $z_{1}$ and $z_{2}$ are either in $\mathbb{Z}$ or in $\mathbb{Z}[\sqrt{g}]$. Looking at the coefficient of $p^{4}$ in the approximation congruence mod $p^{5}$, we infer a condition

$$
d_{0}+d_{1} z_{k}+a_{20} z_{k}^{2} \equiv 0 \quad(\bmod p),
$$

where $d_{0}$ and $d_{1}$ are rational integers determined by $x$ and by the first digits of $a_{0}, \ldots, a_{4}$.

If the solutions $z_{1}$ and $z_{2}$ of the last congruence are distinct, then $v_{p}\left(T_{1}-T_{2}\right)=2$ and Krasner's lemma implies that $T_{k}-x p \in \mathbb{Q}_{p}\left(z_{k}\right)$. Thus, $E / \mathbb{Q}_{p}$ is unramified, and we have $\gamma=v_{p}\left(f_{\theta}^{\prime}\left(T_{k}\right)\right)=2$ by Proposition 3. In this case the algorithm may be started with $t_{k}=x p+z_{k} p^{2}$. 
We found two examples of this kind, for the characters $(3,13564,0)$ and $(3,-11188,1)$, both with $E=\mathbb{Q}_{3}(\sqrt{2})$. They are presented in detail in Table VII.

Secondly, suppose that $z_{1}=z_{2}=z$. Then $z \in \mathbb{Z}$, and one proceeds with the congruence

$$
T_{1,2} \equiv x p+z p^{2} \pm u p^{2} \sqrt{r p} \quad\left(\bmod p^{3}\right)
$$

where $u \in \mathbb{Z}, 0 \leq u<p$. Put $\pi=\sqrt{r p}$. To find $u$ and $r$, one has to solve a congruence of the form $r u^{2} \equiv l(\bmod p)$, where $l \in \mathbb{Z}$; this is in fact obtained from $\mathscr{C}\left(\pi^{11}\right)$. If $u \neq 0$, we see that $E=\mathbb{Q}_{p}(\pi)$ and $v_{p}\left(T_{1}-T_{2}\right)=5 / 2$, and Proposition 3 gives $\gamma=v_{\pi}\left(f_{\theta}^{\prime}\left(T_{k}\right)\right)=5$. Hence, we may take $t_{1,2}=$ $x p+z p^{2} \pm u p^{2} \pi$.

In Table VII, the example for the character $(3,13784,0)$ belongs to this category. Table VIIa shows two similar examples of type $Z$.

If $u=0$, i.e., $v_{p}\left(T_{1}-T_{2}\right) \geq 3$, we are in the analogous situation we started from. There is no example of this in our material.

\section{VARIOUS TYPES OF THE NEWTON POLYGON}

We now show how to deal with some classes of the Newton polygon of $f_{\theta}(T)$ which differ from our two basic types. They are called Types 3-6 below; each of them was met in the computations and will be treated in a generality sufficient to settle our particular examples.

The examples mentioned in this section appear in Table VIII unless otherwise stated.

Type 3. $v_{p}\left(a_{0}\right)>2, v_{p}\left(a_{1}\right)=1, \lambda=2$.

The two zeros $T_{1}$ and $T_{2}$ satisfy $v_{p}\left(T_{1}\right)=v_{p}\left(a_{0}\right)-1>1$ and $v_{p}\left(T_{2}\right)=1$. Hence they lie in $\mathbb{Q}_{p}$. This is a slight variant of our "second type", and from $\S \mathrm{I} .9$ it is seen, on putting $a_{02}=0$, that one may start with $t_{k}=x_{k} p \quad(k=1,2)$, where $x_{1}=0$ and $x_{2}$ is the root of the congruence $a_{11}+a_{20} x \equiv 0(\bmod p)$.

Examples of this kind are obtained for the characters $(5,3101,0),(5,-4371$, $1),(11,-723,1)$, the last two being of type $Z$. The example $(3,-1399,1)$ in Table II (Part I) also belongs to this family.

Type 4. $v_{p}\left(a_{0}\right)=c>1, v_{p}\left(a_{k}\right)>c(1-k / \lambda)$ for $k=1, \ldots, \lambda-1, \lambda(>1)$ prime to $c$ and $p$.

The Newton polygon has one nonzero slope, and this equals $-c / \lambda$. As in the case of the "first type", the zeros $T_{0}\left(=T_{1}, \ldots, T_{\lambda}\right)$ are in fully ramified extensions $E=\mathbb{Q}_{p}(\sqrt[\lambda]{r p})$. Because $c$ and $p$ are prime to $\lambda$, one easily verifies that $\gamma=v_{\pi}\left(\lambda a_{\lambda} T_{0}^{\lambda-1}\right)=(\lambda-1) c$. If $\lambda=2$, one has to solve for $x$ and $r$ the congruence $a_{0 c}+a_{20} r^{c} x^{2} \equiv 0(\bmod p)$, obtained from $\mathscr{C}\left(\pi^{2 c+1}\right)$, and then take $t_{0}=x \pi^{c}$. For $\lambda>2$ the procedure is similar but more complicated.

We have two examples with $c=3$ and $\lambda=2$, namely for $(3,1901,0)$ and $(5,12056,2)$, and one similar example of type $Z$, for $(5,-7816,1)$. Table III in Part I gives an example with $(5,1317,0)$ in which $c=2$ and $\lambda=3$.

Type 5. $v_{p}\left(a_{0}\right)=2, v_{p}\left(a_{1}\right) \geq 2, \lambda=4$.

As in Type 4, there is but one nonzero slope, this time equal to $-1 / 2$. The zeros $T_{1}, \ldots, T_{4}$ lie in fully ramified quartic extensions $E=\mathbb{Q}_{p}(\sqrt[4]{r p})$ or, if this is not the case, either in quadratic or biquadratic extensions of $\mathbb{Q}_{p}$ (here "biquadratic" means "a composite of two quadratic").

An example of the former kind is given by $(3,1541,0)$. The computation 
is analogous to that in Type 4; note that Proposition 3 yields $\gamma=7$ (see Table VIII). On the other hand, for the character $(3,4204,0)$ one rules out the fields $\mathbb{Q}_{3}(\sqrt[4]{ \pm 3})$ by using $\mathscr{C}\left(\pi^{9}\right)$, and it turns out that the zeros are contained in $\mathbb{Q}_{3}(\sqrt{3}, \sqrt{2})$. In this case there is no zero of any $L_{3}\left(s, \theta \psi_{n}\right), n \geq 0$, corresponding to $T_{0}$; indeed, $v_{3}\left(\log \left(1+T_{0}\right)\right)=1 / 2$, and so $v_{3}\left(s_{0}\right)=-1 / 2$.

In these two examples, the approximate values of the zeros given in Table VIII are easily computed by hand. A third example of the same kind, not included in the tables, is $(3,7244,0)$. In this case we have $E=\mathbb{Q}_{3}(\sqrt[4]{-3})$.

Type 6. $v_{p}\left(a_{0}\right) \geq 2, v_{p}\left(a_{1}\right)=1, \lambda \geq 3, \lambda-1$ prime to $p$.

Among the zeros $T_{0}=T_{k}, k=1, \ldots, \lambda$, there is one, say $T_{1}$, belonging to $\mathbb{Q}_{p}$ and satisfying $v_{p}\left(T_{1}\right) \geq v_{p}\left(a_{0}\right)-1 \geq 1$. The other zeros satisfy $v_{p}\left(T_{k}\right)=$ $1 /(\lambda-1)(k=2, \ldots, \lambda)$. For $T_{0}=T_{1}$, Proposition 3 yields

$$
\gamma=\sum_{j=2}^{\lambda} v_{p}\left(T_{1}-T_{j}\right)=\sum_{j=2}^{\lambda} \frac{1}{\lambda-1}=1
$$

Thus, it is sufficient to look at $\mathscr{C}\left(p^{3}\right)$, and this gives $t_{0}=x p$ with $x \equiv-a_{02} / a_{11}$ $(\bmod p)$.

The computation of the remaining zeros is analogous to that in the "first type". In particular, $E / \mathbb{Q}_{p}$ is a fully and tamely ramified extension of degree $\lambda-1$. We find that $v_{p}\left(T_{k}-T_{j}\right)=1 /(\lambda-1)$ for $j=1, \ldots, \lambda, j \neq k$, and so $\gamma=\lambda-1$.

To the first zero $T_{1}$ there always corresponds a zero $s_{1}$ of $L_{p}(s, \theta)$. The same holds true for $T_{2}, \ldots, T_{\lambda}$ in the case $\lambda<p$, while the situation varies for $\lambda \geq p$.

Our examples with $\lambda=3<p$ are the characters $(5,4924,2)$ and $(7,-4072$, $5)$. The character $(3,-25528,1)$ has $\lambda=5$ (computed first by Kobayashi), and $s_{2}, \ldots, s_{5}$ lie outside of $D_{s}$. So also do $s_{2}, s_{3}$ in the example $(3,3512,0)$, where $\lambda=3$. Compare this example with $(3,7804,0)$ from Table VIa: here, too, $\lambda=3$ but $s_{2}$ and $s_{3}$ are zeros of $L_{3}\left(s, \theta \psi_{ \pm}\right)$. Finally, the character $(3,-5051,1)$ in Table $\mathrm{V}$ (Part I), for which $f_{\theta}(T)$ is of type $Z$, also fits this category.

\section{WILDLY RAMIFIED EXTENSIONS}

All examples of wildly ramified extensions $E / \mathbb{Q}_{p}$ were found for $p=3$. The degree of the extensions is 3 or 6 .

Consider first the case of cubic extensions. Let $\lambda=3$ and $v_{3}\left(a_{0}\right)=1$, so that $v_{3}\left(T_{0}\right)=1 / 3$ and $E / \mathbb{Q}_{3}$ is indeed wildly ramified of degree 3 . One may identify $E$ (up to conjugates) by providing a cubic Eisenstein polynomial $r(X) \in \mathbb{Z}_{3}[X]$ such that $E=\mathbb{Q}_{3}(\pi)$ with $r(\pi)=0$. In the table below, the list of 11 such polynomials $r(X)$ corresponds to a complete system of nonconjugate fields $E$. This list is extracted from results by Amano [1], who characterizes in this way all ramified extensions of degree $p$ over any finite extension of $\mathbb{Q}_{p}$.

We will give a further characterization of the fields $E$, better suited for their computational identification. Note that every $r(X)$ is of the form

$$
r(X)=X^{3}-3 a X^{2}-3 b X-3 c,
$$

where $a, b, c \in \mathbb{Z}$ with $c \equiv 1(\bmod 3)$. Let $\pi$ and $E$ be as above. We have $3=\epsilon \pi^{3}$, with $\epsilon$ a unit of $\mathscr{O}_{E}$, and from $r(\pi)=0$ it follows that 
$\epsilon=1 /\left(c+b \pi+a \pi^{2}\right)$. In particular, $\epsilon \equiv 1(\bmod \pi)$. Write

$$
\epsilon=1+\sum_{\nu=1}^{\infty} \epsilon_{\nu} \pi^{\nu}, \quad \epsilon_{\nu} \in\{0, \pm 1\} .
$$

A straightforward calculation shows that our fields $E$ can be identified by the triple $\left(\epsilon_{1}, \epsilon_{2}, \epsilon_{3}\right)$ as indicated in the table.

\begin{tabular}{|l|l|l|}
\hline$E$ & $r(X)$ & $\left(\epsilon_{1}, \epsilon_{2}, \epsilon_{3}\right)$ \\
\hline$A_{1}$ & $X^{3}-3 X-3$ & $(-1,+1,-1)$ \\
$A_{2}$ & $X^{3}+3 X-3$ & $(+1,+1,+1)$ \\
$B_{1}$ & $X^{3}-3 X^{2}-12$ & $(0,-1,-1)$ \\
$B_{2}$ & $X^{3}-3 X^{2}-3$ & $(0,-1,0)$ \\
$B_{3}$ & $X^{3}-3 X^{2}+6$ & $(0,-1,+1)$ \\
& & \\
\hline
\end{tabular}

\begin{tabular}{|l|l|l|}
\hline$E$ & $r(X)$ & $\left(\epsilon_{1}, \epsilon_{2}, \epsilon_{3}\right)$ \\
\hline$C_{1}$ & $X^{3}+3 X^{2}-12$ & $(0,+1,-1)$ \\
$C_{2}$ & $X^{3}+3 X^{2}-3$ & $(0,+1,0)$ \\
$C_{3}$ & $X^{3}+3 X^{2}+6$ & $(0,+1,+1)$ \\
$P_{1}$ & $X^{3}-12$ & $(0,0,-1)$ \\
$P_{2}$ & $X^{3}-3$ & $(0,0,0)$ \\
$P_{3}$ & $X^{3}+6$ & $(0,0,+1)$ \\
\hline
\end{tabular}

Here the fields are labeled with $A_{1}, A_{2}, B_{1}$, etc. The extension $E / \mathbb{Q}_{3}$ is cyclic for $E=C_{1}, C_{2}, C_{3}$ and pure for $E=P_{1}, P_{2}, P_{3}$.

We computed 17 examples in which the zeros belong to the fields of this table; eight of them were selected to be exhibited in Table IX. In the entire sample of 17 fields, $A_{1}$ and $A_{2}$ occur five times each, supporting the natural hypothesis that the values of $\epsilon_{1}$ be randomly distributed.

The functions $L_{3}(s, \theta)$ and $f_{\theta}(T)$ were computed with the parameter $\eta=$ 16. This implies, by Propositions I.12 and I.7, that the coefficients $a_{j}$ are obtained mod $3^{8-j}(j=0, \ldots, 7)$. It is seen below that this accuracy allows us to determine the field $E$ in all examples but one, and to find the zeros

$$
T_{0}=\sum_{\nu=1}^{\infty} x_{\nu} \pi^{\nu} \quad\left(x_{\nu} \in\{0, \pm 1\}, x_{1} \neq 0\right)
$$

to 1-4 $x_{\nu}$-places. We computed $T_{0}$ by hand, thus avoiding the rather tedious implementation of the arithmetic of the fields in question. The results were checked by computing $f_{\theta}\left(T_{0}\right)$. From $\S \mathrm{I} .3$ it follows that the corresponding numbers $s_{0}$ are not zeros of any $L_{3}\left(s, \theta \psi_{n}\right)$; hence they are ignored.

To find $E$ and $T_{0}$, start with the approximation congruence $\mathscr{C}\left(\pi^{5}\right)$. This reduces to the congruences

$$
a_{01}+a_{30} x_{1} \equiv 0, \quad a_{01} \epsilon_{1}+a_{11} x_{1}+a_{40} \equiv 0 \quad(\bmod 3),
$$

which give us $x_{1}$ and $\epsilon_{1}$. If $\epsilon_{1}=-1$ or +1 , the field is $A_{1}$ or $A_{2}$, respectively, and the coefficients $\epsilon_{2}, \epsilon_{3}, \ldots$ are determined by the equation $\epsilon=1 /(1 \pm \pi)$. Better approximations for $T_{0}$ then follow easily from $\mathscr{C}\left(\pi^{m}\right)$ for bigger exponents $m$.

In Table IX, the characters $(3,-3592,1)$ and $(3,1781,0)$ are examples giving the field $A_{1}$. The latter requires slightly different techniques since $v_{3}\left(a_{0}\right)$ $=2$. In both examples, the zeros $T_{1}, T_{2}, T_{3}$ lie in conjugate extensions generated by the roots $\pi=\pi_{k}$ of $r(X)=0 \quad(k=1,2,3)$.

Similarly, the characters $(3,281,0),(3,-311,1)$, and $(3,-2132,1)$ yield examples in which $E=A_{2}$. The first of these is also mentioned in $\S$ I.10, with another normalization of $r(X)$. The second example is of type $Z$, while the third shows a function $f_{\theta}(T)$ with two zeros (one trivial) in $\mathbb{Q}_{3}$.

Here are the other examples of $A_{1}$ and $A_{2}$ computed by us. 
Example 1. For $\pi_{k}$ a zero of $X^{3}-3 X-3 \quad(k=1,2,3)$,

$$
\begin{array}{ll}
(3,1397,0): & T_{k} \equiv \pi_{k}-\pi_{k}^{2}-\pi_{k}^{4}\left(\bmod \pi_{k}^{5}\right), \\
(3,5368,0): & T_{k} \equiv-\pi_{k}-\pi_{k}^{2}-\pi_{k}^{3}+\pi_{k}^{4}\left(\bmod \pi_{k}^{5}\right), \\
(3,6712,0): & T_{k} \equiv-\pi_{k}^{2}+\pi_{k}^{4}\left(\bmod \pi_{k}^{5}\right) .
\end{array}
$$

Example 2. $(3,-2920,1): \pi_{k}$ zero of $X^{3}+3 X-3 \quad(k=1,2,3)$,

$$
T_{k} \equiv \pi_{k}+\pi_{k}^{2}+\pi_{k}^{4} \quad\left(\bmod \pi_{k}^{5}\right) .
$$

Example 3. $(3,-4184,1): T_{1}=0, s_{1}=0$,

$$
\begin{aligned}
& \pi_{k} \text { zero of } X^{3}+3 X-3 \quad(k=2,3,4), \\
& T_{k} \equiv \pi_{k}-\pi_{k}^{2}+\pi_{k}^{3}\left(\bmod \pi_{k}^{4}\right) .
\end{aligned}
$$

If $\epsilon_{1}=0$, we compute $\epsilon_{2}$ from the congruence

$$
-a_{01} \epsilon_{2} \equiv a_{50} x_{1}+a_{21} \quad(\bmod 3)
$$

which is a consequence of $\mathscr{C}\left(\pi^{6}\right)$.

Let first $\epsilon_{2}=1$, so that $E / \mathbb{Q}_{3}$ is cyclic. Then $\mathscr{C}\left(\pi^{7}\right)$ gives $\epsilon_{3}$ and so fixes $E$. When computing further places for $T_{0}$, one should observe that $E$ now contains all the three zeros of $f_{\theta}(T)$.

We have two examples of cyclic fields, in fact, of $E=C_{1}$. One, included in Table IX, is for $(3,2504,0)$, the other is given below.

Example 4. $(3,5624,0): \pi$ zero of $X^{3}+3 X^{2}-12$,

$$
T_{1} \equiv-\pi-\pi^{3}, \quad T_{2} \equiv-\pi+\pi^{2}-\pi^{3}, \quad T_{3} \equiv-\pi-\pi^{2} \quad\left(\bmod \pi^{4}\right) .
$$

Secondly, suppose that $\epsilon_{2}=0$. Then $E$ is a pure extension of $\mathbb{Q}_{3}$ and one finds $\epsilon_{3}$ and $x_{2}$ by solving simultaneously a pair of congruences produced by $\mathscr{C}\left(\pi^{8}\right)$.

In Table IX, this case is represented by the characters $(3,4172,0)$ and $(3,-1144,1)$, which lead to the fields $\mathbb{Q}_{3}(\sqrt[3]{12})$ and $\mathbb{Q}_{3}(\sqrt[3]{-6})$, respectively. The next two examples give our other characters of this kind. Example 6 introduces a case with $\lambda=4$; here the ultimate identification of $E$ must be left open.

Example 5. For $\pi_{k}$ a zero of $X^{3}+6(k=1,2,3)$,

$$
\begin{array}{ll}
(3,401,0): & T_{k} \equiv-\pi_{k}+\pi_{k}^{2}\left(\bmod \pi_{k}^{3}\right), \\
(3,4472,0): & T_{k} \equiv \pi_{k}+\pi_{k}^{2}\left(\bmod \pi_{k}^{3}\right) .
\end{array}
$$

Example 6. $(3,6856,0): T_{1}=0.2201, s_{1}=2.000$,

$$
\begin{aligned}
& \pi_{k} \text { zero of } X^{3}-a(k=2,3,4) \text { with } a=4,1, \\
& \text { or }-2, \\
& T_{k} \equiv \pi_{k}\left(\bmod \pi_{k}^{2}\right) .
\end{aligned}
$$

After this, it is also clear how to deal with $\epsilon_{2}=-1$ corresponding to the fields $B_{1}, B_{2}, B_{3}$. We have no example of these fields.

Now let us turn to the case of sextic extensions. The basic case is the one with $\lambda=6$ and $v_{3}\left(a_{0}\right)=1$. As usual, put $E=\mathbb{Q}_{3}\left(T_{0}\right)=\mathbb{Q}_{3}(\pi)$. 
Proposition 4. For a fully ramified sextic extension $E=\mathbb{Q}_{3}(\pi)$, let $\epsilon$ be the unit of the ring $\mathscr{O}_{E}$ defined by the equation $3=\epsilon \pi^{6}$. The field $E$ contains the subfield $\mathbb{Q}_{3}(\sqrt{3})$ or $\mathbb{Q}_{3}(\sqrt{-3})$ according as $\epsilon \equiv 1$ or $\epsilon \equiv-1(\bmod \pi)$, respectively.

Proof. The polynomial $q(X)=X^{2} \mp 3$ satisfies $v_{\pi}\left(q^{\prime}\left(\pi^{3}\right)\right)=3$ and $q\left(\pi^{3}\right)=$ $\pi^{6}(1 \mp \epsilon) \equiv 0\left(\bmod \pi^{7}\right)$ provided that $\epsilon \equiv \pm 1(\bmod \pi)$.

It follows that $E$ is a ramified cubic extension of $\mathbb{Q}_{3}(\sqrt{3})$ or $\mathbb{Q}_{3}(\sqrt{-3})$. Hence, it can be classified in the way shown by Amano in [1]. We do not write down the complete classification but, rather, show how to handle the specific examples we have of this case. They are the three examples presented in Table $\mathrm{X}$, two of them with $\lambda=6$ and one with $\lambda=7$ and $f_{\theta}(T)$ of type $Z$. In these examples we chose the truncation parameter $\eta=18$ or 20 according as $\lambda=6$ or 7 , respectively. This enables us to identify $E$ and compute $T_{0} \bmod \pi^{3}$. As mentioned in $\S 2$, we never have $E=\mathbb{Q}_{3}\left(\zeta_{9}\right)$; hence, we do not compute $s_{0}$.

Put $\rho=\sqrt{(-1)^{z} 3}$, where $z=0$ or 1 . By Proposition $4, E=\mathbb{Q}_{3}(\rho, \pi)$, where $\pi$ is a zero of an Eisenstein polynomial $r(X) \in \mathbb{Q}_{3}(\rho)[X]$. From [1] we find that it suffices to consider the polynomials

$$
r(X)=X^{3}-a \rho X^{2}-b \rho X-c \rho,
$$

where $a, b, c \in \mathbb{Z}$ and $c \equiv 1(\bmod \rho)$. Let $\kappa$ be the unit in $\mathbb{Q}_{3}(\rho)$ such that $\rho=\kappa \pi^{3}$. A similar argument as before yields $\kappa=1 /\left(c+b \pi+a \pi^{2}\right)$, and we may write

$$
\kappa=1+\sum_{\nu=1}^{\infty} \kappa_{\nu} \pi^{\nu}, \quad \kappa_{\nu} \in\{0, \pm 1\} .
$$

With the usual notation $T_{0}=\sum_{\nu=1}^{\infty} x_{\nu} \pi^{\nu}\left(\right.$ where $\left.x_{1} \neq 0\right)$ we get from $\mathscr{C}\left(\pi^{8}\right)$ the congruences

$$
(-1)^{z} a_{01}+a_{60} \equiv 0, \quad-2 a_{01} \kappa_{1} \equiv\left(a_{11}+(-1)^{z} a_{70}\right) x_{1} \quad(\bmod 3) .
$$

The former determines $\rho$, and the latter tells us, first, whether or not $\kappa_{1}=0$. If $\kappa_{1} \neq 0$-as is the case in our examples-then $b \neq 0$, and we arrive, by [1], at the polynomials

$$
r(X)=X^{3} \mp \rho X-\rho,
$$

which generate six ramified extensions of $\mathbb{Q}_{3}(\rho)$. The six zeros of $f_{\theta}(T)$ are contained in these fields, one in each. It also follows that $\kappa=1 /(1 \pm \pi)$ and so, in particular, $\kappa_{2}=1$. Hence the approximation congruence $\mathscr{C}\left(\pi^{9}\right)$ enables us to compute $x_{2}$.

In case one is interested just in knowing whether or not $E=\mathbb{Q}_{3}\left(\zeta_{9}\right)$, it is worth noting that (4) yields the following necessary conditions for this equality: $a_{01}=a_{60}, a_{11}=a_{70}$. Indeed, we have $z=1$ and $\kappa_{1}=0$ in this case.

In our examples obtained for the characters $(3,-7108,1)$ and $(3,-20692$, 1 ), the first congruence in (4) gives the result $z=0$ and $z=1$, respectively, and the second gives $\kappa_{1}=x_{1}$. For the third character $(3,-1832,1)$ we find that $z=0$ and $\kappa_{1}=-x_{1}$. See Table $\mathrm{X}$ for the full result. 
TABLE VI. $\lambda=2, a_{0} \neq 0, s_{1,2}$ zeros of $L_{p}\left(s, \theta \psi_{ \pm}\right)$

\begin{tabular}{|l|l|l|}
\hline \multicolumn{3}{|c|}{$(3,653,0)$} \\
\hline 0.21100 & $0.0200 \pm 0.0000 \pi$ & 0.21100 \\
0.00102 & $0.0022 \pm 0.1111 \pi$ & 0.0202 \\
0.02001 & $0.0222 \pm 0.0011 \pi$ & 2.212 \\
0.00022 & $0.0002 \pm 0.0211 \pi$ & 1.01 \\
0.00220 & $0.0021 \pm 0.0002 \pi$ & 2.0 \\
0.00002 & $0.0000 \pm 0.0001 \pi$ & 2. \\
0.00020 & $0.0002 \pm 0.0000 \pi$ & \\
\hline \multicolumn{3}{|c|}{$=\sqrt{-3}$} \\
$T_{1.2}=0.12 \pm 2.2 \pi$ \\
$s_{1,2}=0.0 \pm(2.) \pi$ \\
\hline \hline \multicolumn{3}{|c|}{$(3,-379,1)$} \\
\hline 0.20000 & $0.0000 \pm 0.1100 \pi$ & 0.20000 \\
0.00111 & $0.0002 \pm 0.2212 \pi$ & 0.0121 \\
0.02122 & $0.0200 \pm 0.0022 \pi$ & 2.210 \\
0.00112 & $0.0012 \pm 0.0101 \pi$ & 2.21 \\
0.00200 & $0.0022 \pm 0.0002 \pi$ & 1.1 \\
0.00002 & $0.0000 \pm 0.0002 \pi$ & 0. \\
0.00021 & $0.0002 \pm 0.0000 \pi$ & \\
\hline \multicolumn{3}{|c|}{$=\sqrt{-3}$} \\
$T_{1,2}=0.20 \pm 2.1 \pi$ \\
$s_{1,2}=1.0 \pm(1.) \pi$ & \\
\hline
\end{tabular}

\begin{tabular}{|l|l|l|}
\hline \multicolumn{3}{|c|}{$(3,1153,0)$} \\
\hline 0.11000 & $0.0110 \pm 0.0000 \pi$ & 0.11000 \\
0.00210 & $0.0010 \pm 0.1011 \pi$ & 0.0202 \\
0.01002 & $0.0111 \pm 0.0010 \pi$ & 1.202 \\
0.00012 & $0.0001 \pm 0.0212 \pi$ & 2.01 \\
0.00121 & $0.0010 \pm 0.0002 \pi$ & 0.1 \\
0.00001 & $0.0000 \pm 0.0001 \pi$ & 2. \\
0.00012 & $0.0001 \pm 0.0000 \pi$ & \\
\hline \multicolumn{3}{|c|}{$=\sqrt{-3}$} \\
$T_{1,2}=0.12 \pm 2.2 \pi$ \\
$s_{1,2}=0.0 \pm(1.) \pi$ \\
\hline \hline \multicolumn{3}{|c|}{$(3,-1336,1)$} \\
\hline 0.22000 & $0.0100 \pm 0.2100 \pi$ & 0.22000 \\
0.00102 & $0.0020 \pm 0.1220 \pi$ & 0.0100 \\
0.02212 & $0.0210 \pm 0.0001 \pi$ & 2.200 \\
0.00122 & $0.0010 \pm 0.0200 \pi$ & 2.00 \\
0.00210 & $0.0020 \pm 0.0002 \pi$ & 0.1 \\
0.00000 & $0.0000 \pm 0.0001 \pi$ & 0. \\
0.00022 & $0.0002 \pm 0.0000 \pi$ & \\
\hline \multicolumn{3}{|c|}{$=\sqrt{-3}$} \\
$T_{1,2}=0.20 \pm 2.0 \pi$ \\
$s_{1,2}=1.0 \pm(0.) \pi$ \\
\hline
\end{tabular}

TABLE VIa. $\lambda=3, s_{2,3}$ zeros of $L_{p}\left(s, \theta \psi_{ \pm}\right)$

\begin{tabular}{|l|l|l|}
\hline \multicolumn{3}{|c|}{$(3,-827,1)$} \\
\hline 0 & $0.0200 \pm 0.0122 \pi$ & 0 \\
0.012222 & $0.0112 \pm 0.0222 \pi$ & 0.200011 \\
0.001201 & $0.0010 \pm 0.0021 \pi$ & 0.02011 \\
0.000021 & $0.0000 \pm 0.0010 \pi$ & 2.2100 \\
0.000120 & $0.0001 \pm 0.0001 \pi$ & 0.022 \\
0.000010 & & 2.02 \\
0.000011 & & 1.2 \\
0.000001 & & 1. \\
\hline \multicolumn{2}{|c|}{$T_{1}=0}$, & $s_{1}=0$ \\
$\pi$ & $=\sqrt{-3}$ & \\
$T_{2,3}$ & $=0.0000 \pm 2.210 \pi$ \\
$s_{2,3}$ & $=1.011 \pm 0.11 \pi$ \\
\hline
\end{tabular}

\begin{tabular}{|c|c|c|}
\hline \multicolumn{3}{|c|}{$(3,7804,0)$} \\
\hline $\begin{array}{l}0.01100 \\
0.01220 \\
0.00000 \\
0.00020 \\
0.00021 \\
0.00000 \\
0.00001\end{array}$ & $\begin{array}{l}0.0020 \pm 0.0212 \pi \\
0.0120 \pm 0.0222 \pi \\
0.0000 \pm 0.0001 \pi \\
0.0002 \pm 0.0021 \pi \\
0.0002 \pm 0.0000 \pi\end{array}$ & $\begin{array}{l}0.01100 \\
0.1010 \\
0.112 \\
1.21 \\
2.2 \\
2 .\end{array}$ \\
\hline $\begin{array}{l}T_{1}=0 \\
\pi \\
T_{2,3} \\
s_{2,3}\end{array}$ & $\begin{array}{l}s_{1}=2.001 \\
=\sqrt{-3} \\
=0.1 \pm 2.0 \pi \\
=(0 .) \pm(1 .) \pi\end{array}$ & \\
\hline
\end{tabular}


TABLE VII. $\lambda=2, \quad a_{0} \neq 0, \quad v_{p}\left(T_{1}-T_{2}\right) \geq 2$

\begin{tabular}{|l|l|}
\hline \multicolumn{2}{|c|}{$(3,13564,0)$} \\
\hline 0.011000 & 0.0110000 \\
0.010111 & 0.112210 \\
0.012011 & 1.21101 \\
0.001102 & 1.1101 \\
0.001210 & 1.221 \\
0.000010 & 1.02 \\
0.000101 & 1.1 \\
0.00000 & 0. \\
0.000002 & \\
0.000002 & \\
0.000002 & \\
\hline \multicolumn{2}{|c|}{$=\sqrt{2}$} \\
$T_{1,2}=0.10201 \pm 0.01110 \xi$ \\
$s_{1,2}=1.0111 \pm 0.1120 \xi$ \\
\hline \multicolumn{2}{|l}{} \\
\hline
\end{tabular}

\begin{tabular}{|l|l|}
\hline \multicolumn{2}{|c|}{$(3,13784,0)$} \\
\hline 0.022000 & 0.0220000 \\
0.011110 & 0.222100 \\
0.020221 & 2.11112 \\
0.001212 & 2.0212 \\
0.002001 & 0.121 \\
0.000010 & 0.02 \\
0.000221 & 2.2 \\
0.000000 & 2. \\
0.000001 & \\
0.000002 & \\
0.000001 & \\
\hline \multicolumn{2}{|c|}{$=\sqrt{3}$} \\
$T_{1,2}=0.10122 \pm 0.0112 \pi$ \\
$s_{1,2}=2.1221 \pm 0.201 \pi$ \\
\hline
\end{tabular}

\begin{tabular}{|l|l|}
\hline \multicolumn{2}{|c|}{$(3,-11188,1)$} \\
\hline 0.011000 & 0.01100000 \\
0.011000 & 0.1222201 \\
0.010000 & 1.012021 \\
0.002112 & 2.12101 \\
0.001021 & 1.2000 \\
0.000010 & 2.100 \\
0.000112 & 0.12 \\
0.000002 & 0.1 \\
0.000002 & 0. \\
0.000001 & \\
0.000002 & \\
\hline \multicolumn{2}{|c|}{$=\sqrt{2}$} \\
$T_{1,2}=0.102122 \pm 0.012001 \xi$ \\
$s_{1,2}=1.01000 \pm 0.12121 \xi$ \\
\hline
\end{tabular}

TABLE VIIa. $\lambda=3, a_{0}=0, \quad v_{p}\left(T_{2}-T_{3}\right) \geq 2$

\begin{tabular}{|l|l|}
\hline \multicolumn{2}{|c|}{$(3,-2564,1)$} \\
\hline 0 & 0 \\
0.00201120 & 0.0122220 \\
0.00200201 & 0.211210 \\
0.00211101 & 1.00101 \\
0.00001112 & 1.2000 \\
0.00000110 & 2.012 \\
0.00001222 & 1.20 \\
0.00000112 & 1.2 \\
0.00000010 & 1. \\
0.00000100 & \\
0.00000001 & \\
0.00000000 & \\
0.00000001 & \\
\hline$T_{1}=0$, & $s_{1}=0$ \\
$\pi$ & $=\sqrt{2 \cdot 3}$ \\
$T_{2,3}$ & $=0.20112 \pm 0.0101 \pi$ \\
$s_{2,3}$ & $=1.2022 \pm 0.210 \pi$ \\
\hline
\end{tabular}

\begin{tabular}{|l|l|}
\hline \multicolumn{2}{|c|}{$(3,-8804,1)$} \\
\hline 0 & 0 \\
0.00101202 & 0.0211122 \\
0.00220021 & 0.222110 \\
0.00111021 & 2.11102 \\
0.00021121 & 0.0100 \\
0.00002121 & 0.120 \\
0.00002022 & 0.21 \\
0.00000111 & 1.0 \\
0.00000010 & 0. \\
0.00000212 & \\
0.00000012 & \\
0.00000001 & \\
0.00000002 & \\
\hline \multicolumn{2}{|c|}{$T_{1}=0, s_{1}=0$} \\
$\pi \quad \sqrt{3}$ \\
$T_{2,3}=0.10001 \pm 0.0102 \pi$ \\
$s_{2,3}=2.2121 \pm 0.220 \pi$ \\
\hline
\end{tabular}


TABLE VIII. Various types of the Newton polygon

\begin{tabular}{|l|l|}
\hline \multicolumn{2}{|c|}{$(5,3101,0)$} \\
\hline 0.004012 & 0.0040120 \\
0.040113 & 0.423301 \\
0.031110 & 3.21012 \\
0.004403 & 1.4114 \\
0.000342 & 1.234 \\
0.000013 & 1.31 \\
0.000042 & 4.2 \\
0.000003 & 0. \\
\hline \multicolumn{2}{|c|}{$T_{1}=0.040243$} \\
$s_{1}=0.42140$ \\
$T_{2}=0.224113$ \\
$s_{2}=2.12443$ \\
\hline \multicolumn{2}{|c}{}
\end{tabular}

\begin{tabular}{|l|l|}
\hline \multicolumn{2}{|c|}{$(5,-4371,1)$} \\
\hline 0 & 0 \\
0.000303 & 0.003204 \\
0.003234 & 0.31301 \\
0.003302 & 3.1142 \\
0.000003 & 3.404 \\
0.000021 & 1.30 \\
0.000003 & 4.1 \\
0.000001 & 0. \\
\hline \multicolumn{2}{|c|}{$=0, s_{1}=0$} \\
$T_{2}=0.04102$ \\
$s_{2}=0.4240$ \\
$T_{3}=0.44340$ \\
$s_{3}=4.2101$ \\
\hline
\end{tabular}

\begin{tabular}{|l|l|}
\hline \multicolumn{2}{|c|}{$(11,-723,1)$} \\
\hline 0 & 0 \\
$0.0009 \alpha \alpha \alpha$ & 0.0082679 \\
$0.008 \alpha 109$ & 0.745759 \\
0.0062906 & 1.47320 \\
$0.00084 \alpha 3$ & 6.7683 \\
0.0000657 & 0.116 \\
0.0000028 & 8.70 \\
0.0000006 & 1.3 \\
\hline \multicolumn{2}{|c|}{$=0, s_{1}=0$} \\
$T_{1}=0.0246697$ \\
$T_{2}=0.33543 \alpha$ \\
$s_{2}=0.42 \alpha 5014$ \\
$T_{3}=6.7852 \alpha 0$ \\
$s_{3}=6$
\end{tabular}

\begin{tabular}{|l|l|}
\hline \multicolumn{2}{|c|}{$(3,1901,0)$} \\
\hline 0.0020 & 0.0020 \\
0.0012 & 0.022 \\
0.0120 & 1.11 \\
0.0010 & 1.0 \\
0.0011 & 0. \\
0.0000 & \\
0.0001 & \\
\hline \multicolumn{2}{|c|}{$=\sqrt{3}$} \\
$T_{1,2}=0.020 \pm 0.11 \pi$ \\
$s_{1,2}=0.12 \pm 2.0 \pi$ \\
\hline
\end{tabular}

\begin{tabular}{|l|l|}
\hline \multicolumn{2}{|c|}{$(5,12056,2)$} \\
\hline 0.0042412 & 0.0042412 \\
0.0004221 & 0.004001 \\
0.0134244 & 1.22140 \\
0.0021304 & 1.1110 \\
0.0000210 & 0.112 \\
0.0000002 & 1.21 \\
0.0000302 & 4.2 \\
0.0000043 & 1. \\
\hline \multicolumn{2}{|c|}{$=\sqrt{5}$} \\
$T_{1,2}=0.000443 \pm 0.10420 \pi$ \\
$s_{1,2}=0.02023 \pm 1.2221 \pi$ \\
\hline
\end{tabular}

\begin{tabular}{|l|l|}
\hline \multicolumn{2}{|c|}{$(5,-7816,1)$} \\
\hline 0 & 0 \\
0.0002022 & 0.002044 \\
0.0001311 & 0.01232 \\
0.0030211 & 3.0030 \\
0.0003443 & 1.132 \\
0.0000320 & 1.30 \\
0.0000011 & 2.3 \\
0.0000013 & 3. \\
0.0000003 & \\
\hline$T_{1}=$ & $0, s_{1}=0$ \\
$\pi$ & $=\sqrt{5}$ \\
$T_{2.3}$ & $=0.04202 \pm 0.1211 \pi$ \\
$s_{2,3}$ & $=0.4414 \pm 1.230 \pi$ \\
\hline
\end{tabular}

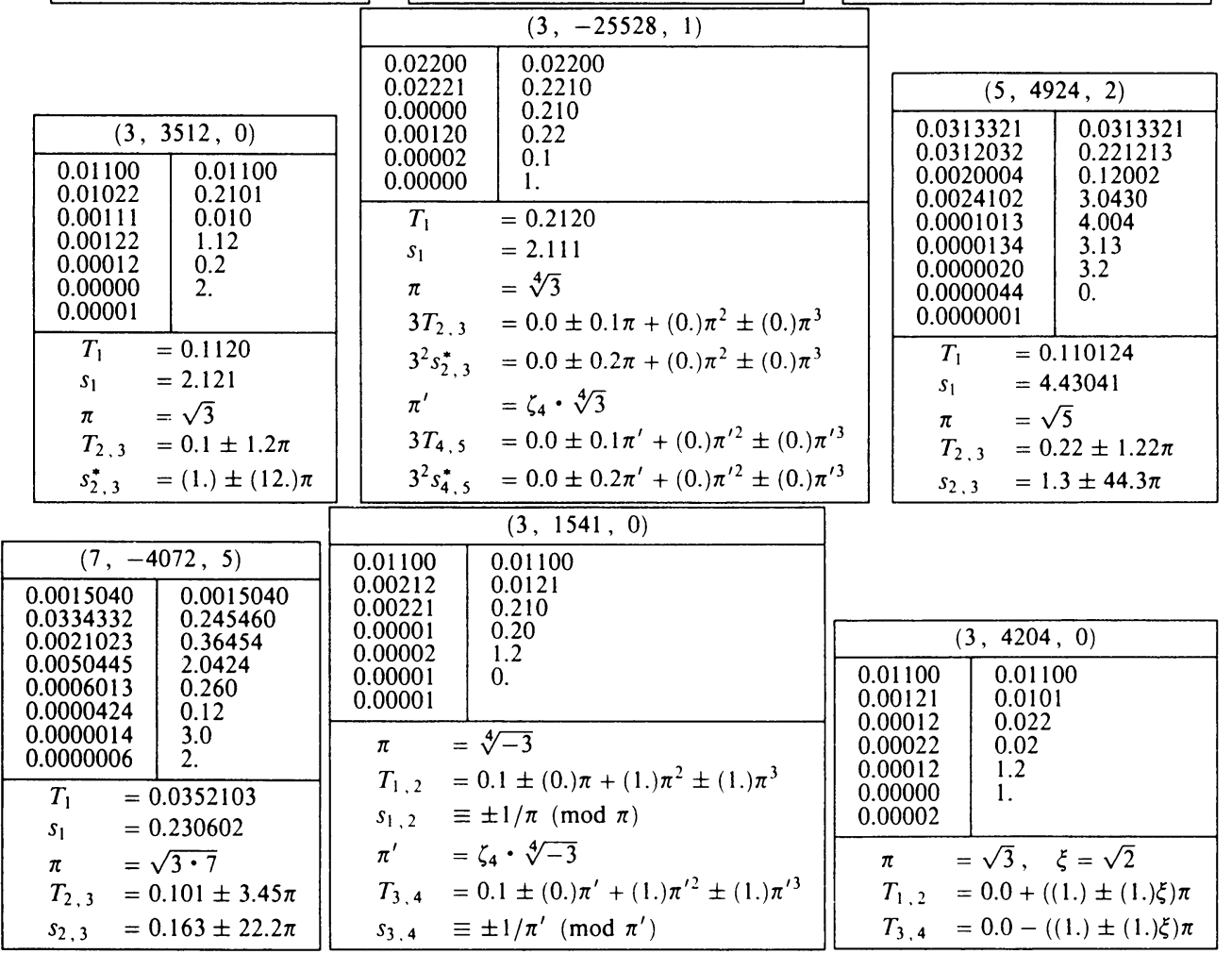


TABLE IX. Zeros in wildly ramified cubic extensions

\begin{tabular}{|c|c|c|}
\hline \multicolumn{2}{|c|}{$(3,-3592,1)$} & $(3$ \\
\hline $\begin{array}{l}0.2200000 \\
0.0221120 \\
0.0020012 \\
0.0002221 \\
0.0000201 \\
0.0000000 \\
0.0000121 \\
0.0000002 \\
0.0000001 \\
0.0000001\end{array}$ & $\begin{array}{l}0.2200000 \\
0.212102 \\
0.12201 \\
2.1012 \\
1.201 \\
1.20 \\
2.0 \\
0 .\end{array}$ & $\begin{array}{l}0.0110000 \\
0.0011021 \\
0.0020221 \\
0.0020210 \\
0.0002012 \\
0.0000011 \\
0.0000222 \\
0.0000010 \\
0.0000001 \\
0.0000011 \\
0.0000001\end{array}$ \\
\hline \multicolumn{2}{|c|}{$\begin{array}{l}\pi_{k} \text { zero of } X^{3}-3 X-3 \\
(k=1,2,3) \\
T_{k} \equiv-\pi_{k}-\pi_{k}^{2}-\pi_{k}^{3}+\pi_{k}^{4} \\
\left(\bmod \pi_{k}^{5}\right)\end{array}$} & $\begin{array}{l}\pi_{k} \text { zero of } \\
(k=1,2 \\
T_{k} \equiv-\pi_{k}^{2} \\
\left(\bmod \pi_{k}^{5}\right)\end{array}$ \\
\hline \multicolumn{2}{|c|}{$(3,2504,0)$} & $(3$, \\
\hline $\begin{array}{l}0.2200000 \\
0.0120012 \\
0.0012201 \\
0.0010020 \\
0.0002102 \\
0.0000220 \\
0.0000001 \\
0.0000012 \\
0.0000002 \\
0.0000022 \\
0.0000001\end{array}$ & $\begin{array}{l}0.2200000 \\
0.220202 \\
0.00020 \\
1.1112 \\
1.112 \\
1.02 \\
2.2 \\
1 .\end{array}$ & $\begin{array}{l}0.1210000 \\
0.0200111 \\
0.0011020 \\
0.0022221 \\
0.0000022 \\
0.0000212 \\
0.0000221 \\
0.0000001 \\
0.0000002 \\
0.0000011\end{array}$ \\
\hline \multicolumn{2}{|c|}{$\begin{array}{l}\pi \text { zero of } X^{3}+3 X^{2}-12, \\
T_{1} \equiv \pi-\pi^{3} \\
T_{2} \equiv \pi+\pi^{2}+\pi^{3}, \\
T_{3} \equiv \pi-\pi^{2}-\pi^{3}\left(\bmod \pi^{4}\right)\end{array}$} & $\begin{array}{l}\pi_{k} \text { zero } \\
(k=1 \\
T_{k} \equiv \pi_{k} \\
\left(\bmod \pi_{k}^{3}\right.\end{array}$ \\
\hline & \multicolumn{2}{|c|}{$(3,-311,1)$} \\
\hline & $\begin{array}{l}0 \\
0.0212121 \\
0.0021022 \\
0.0012211 \\
0.0001120 \\
0.0000202 \\
0.0000020 \\
0.0000011 \\
0.0000001 \\
0.0000020 \\
0.0000002\end{array}$ & $\begin{array}{l}0 \\
0.102010 \\
0.02200 \\
0.2212 \\
2.102 \\
2.22 \\
1.0 \\
0 .\end{array}$ \\
\hline & \multicolumn{2}{|c|}{$\begin{array}{l}T_{1}=0, s_{1}=0 \\
\pi_{k} \text { zero of } X^{3}+3 X-3 \\
(k=2,3,4) \\
T_{k} \equiv \pi_{k}+\pi_{k}^{2}+\pi_{k}^{3} \\
\left(\bmod \pi_{k}^{4}\right)\end{array}$} \\
\hline
\end{tabular}

\begin{tabular}{|l|l|}
\hline \multicolumn{2}{|c|}{$(3,281,0)$} \\
\hline 0.2000000 & 0.2000000 \\
0.0200122 & 0.100111 \\
0.0010010 & 0.20200 \\
0.0001011 & 1.1011 \\
0.0001002 & 0.020 \\
0.0000221 & 1.22 \\
0.0000121 & 1.0 \\
0.0000021 & 2. \\
0.0000002 & \\
0.0000001 & \\
0.0000002 & \\
\hline$\pi_{k}$ zero of $X^{3}+3 X-3$ \\
$(k=1,2,3)$, \\
$T_{k} \equiv \pi_{k}+\pi_{k}^{3}+\pi_{k}^{4}$ \\
$\left(\bmod \pi_{k}^{5}\right)$ \\
\hline \multicolumn{2}{|c|}{$(3,-1144,1)$} \\
\hline 0.2200000 & 0.2200000 \\
0.0210200 & 0.201001 \\
0.0002002 & 0.21221 \\
0.0001222 & 2.1120 \\
0.0002010 & 2.111 \\
0.0000010 & 2.11 \\
0.0000111 & 1.1 \\
0.0000001 & 2. \\
0.0000000 & \\
0.0000002 & \\
0.0000001 & \\
\hline \multicolumn{2}{|c|}{$\pi_{k}$ zero of $X^{3}+6$} \\
$(k=1,2,3)$, \\
$T_{k} \equiv-\pi_{k}+\pi_{k}^{2}$ \\
$\left(\bmod \pi_{k}^{3}\right)$ \\
\hline
\end{tabular}

\begin{tabular}{|l|l|}
\hline \multicolumn{2}{|c|}{$(3,-2132,1)$} \\
\hline 0 & 0 \\
0.0011001 & 0.021101 \\
0.0022202 & 0.21102 \\
0.0001012 & 0.2121 \\
0.0002112 & 0.001 \\
0.0000222 & 1.11 \\
0.0000221 & 2.0 \\
0.0000021 & 0. \\
0.0000001 & \\
0.0000001 & \\
0.0000001 & \\
\hline \multicolumn{2}{|c|}{$T_{1}=0, s_{1}=0$} \\
$T_{2}=0.211, s_{2}=1.12$ \\
$\pi_{k}$ zero of $X^{3}+3 X-3$ \\
$(k=3,4,5)$, \\
$T_{k} \equiv \pi_{k}\left(\bmod \pi_{k}^{3}\right)$ \\
\hline
\end{tabular}


TABLE X. Zeros in wildly ramified sextic extensions

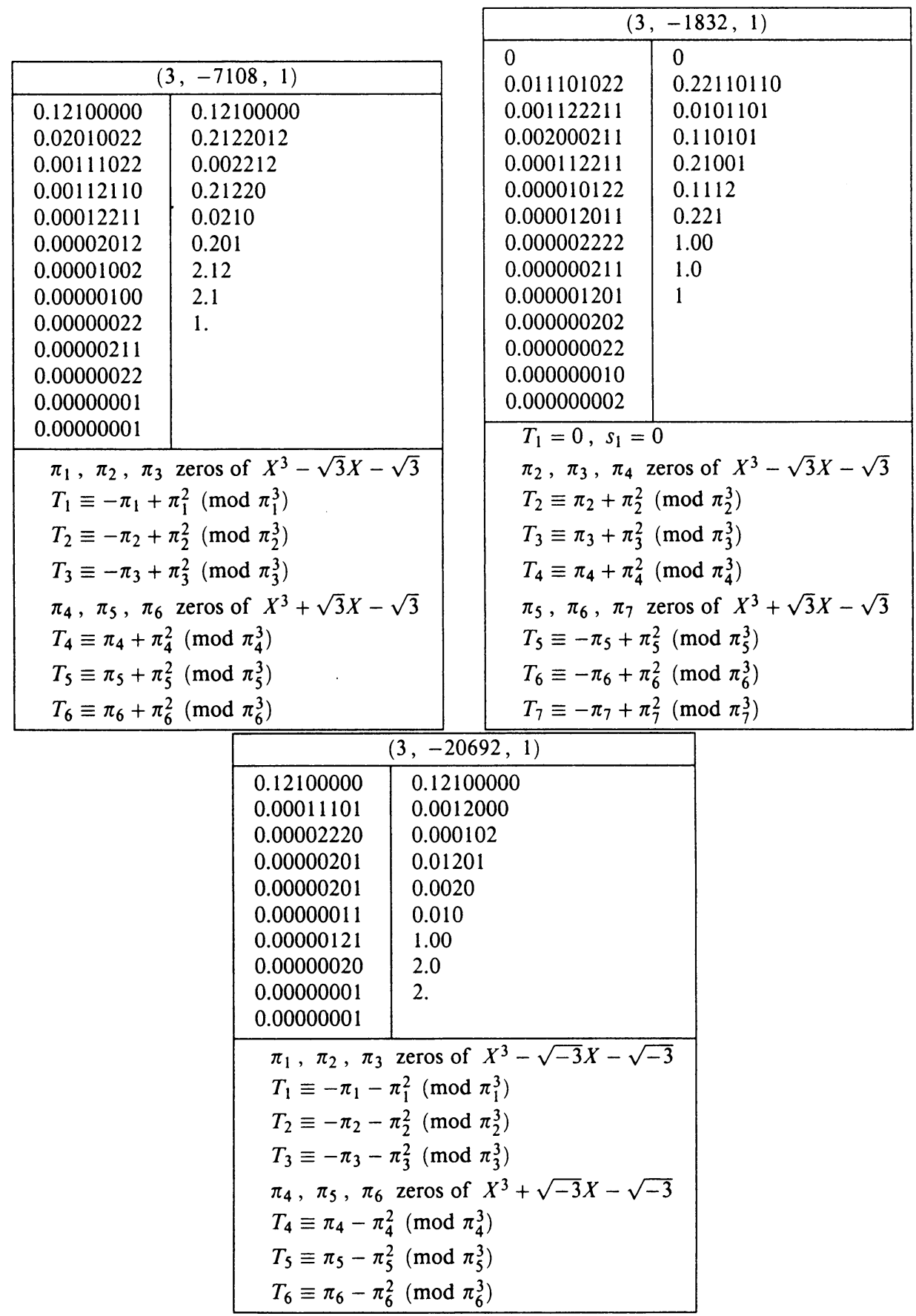




\section{BIBLIOGRAPHY}

1. S. Amano, Eisenstein equations of degree $p$ in a $\mathfrak{p}$-adic field, J. Fac. Sci. Univ. Tokyo Sect. 1A Math. 18 (1971), 1-21.

2. R. Ernvall and T. Metsänkylä, Computation of the zeros of p-adic L-functions, Math. Comp. 58 (1992), 815-830; Supplement, S37-S53.

3. L. C. Washington, Introduction to cyclotomic fields, Springer, Berlin and New York, 1982.

Department of Mathematics, University of Turku, SF-20500 Turku, Finland

E-mail address: taumets@sara.cc.utu.fi 